\title{
Formulation and Solid-State Characterisation of a Novel Benzanilide Co-crystal of the Anti-HIV Drug Efavirenz
}

\author{
Maryam Amra Jordaan and Michael Shapi \\ Faculty of Natural Science, Department of Chemistry, Mangosuthu University of Technology, 511 Mangosuthu Highway, Durban \\ 4000, South Africa
}

\begin{abstract}
HIV/AIDS has had a crippling socio-economic effect on developing countries in Africa. EFV (Efavirenz) is generally used to treat this pathology. However, its clinical efficacy is reduced by its limited water solubility. Our recent efforts were to discover new multicomponent molecular crystals of EFV to further enhance the solubility of EFV, which has led to the formulation of a novel BZA (benzanilide) CC (co-crystal). The molecular complex was characterized by X-ray Powder Diffraction (XRD) and FTIR (Fourier transform infrared) spectroscopy. FTIR data showed a shift in the N-H stretch of the amide group of EFV from $3,312.39 \mathrm{~cm}^{-1}$ to $3,299.71 \mathrm{~cm}^{-1}$ and the $\mathrm{C}=\mathrm{O}$ frequency from $1,749.70 \mathrm{~cm}^{-1}$ to $1,739.87 \mathrm{~cm}^{-1}$ suggesting that the $\mathrm{N}-\mathrm{H}$ and $\mathrm{C}=\mathrm{O}$ functionalities of EFV are participating in hydrogen bonding with the $\mathrm{N}-\mathrm{H}$ and $\mathrm{C}=\mathrm{O}$ moieties of BZA in the EFV-BZA complex, indicative of improved water solubility. Both the XRD patterns of BZA and EFV show characteristic peaks, however, in the XRD pattern of EFV-BZA CC, one unique peak appeared which is absent in both EFV and BZA at $30.8005^{\circ}$ (100\% intensity) suggesting the formation of a new solid phase. The results from this study are significant in understanding the complexity of these scarce EFV based CCs and can be used to advance the development of new solid-state structures with improved physicochemical properties.
\end{abstract}

Key words: CCs, EFV, BZA, X-ray Powder Diffraction (XRD), FTIR.

\section{Introduction}

The onset of HIV/AIDS has led to a despairing situation of disease progression affecting millions of people globally with Africa as its most vulnerable target. EFV (Efavirenz), commercially known as Sustiva ${ }^{\circledR}$ or Stocrin ${ }^{\circledR}$, is a first-line antiretroviral treatment for HIV/AIDS and is categorized as a class 2 compound on the BCS (biopharmaceutical classification system) (i.e. low aqueous solubility but high lipophility and permeability). The low water solubility subsequently, reduces oral bioavailability leading to decreased clinical efficacy. During the past decade studies have centred on the development of multi-component pharmaceutical CCs (co-crystals). Multi-component crystals e.g. solvates, hydrates, CCs and salts play important roles in designing new solids

Corresponding author: Maryam Amra Jordaan, Ph.D., doctor, research fields: medicinal chemistry, photochemistry and organic chemistry. particularly in the pharmaceutical industry. Pharmaceutical CC formation represents a straightforward way to directly influence the solid-state properties of a drug substance, particularly its solubility and hence bioavailability. CCs have regained attention as attractive alternate solid forms for drug development to improve physiochemical properties of pharmaceuticals. New opportunities for producing a larger diversity of solid forms of drug substances exhibiting the proper balance of important properties for development into a viable and effective drug product may be met by CCs [1-3]

CCs used in the pharmaceutical industry are defined as complex crystals formed by a reaction between an API (active pharmaceutical ingredient) and a CCF (co-crystal former); unlike salts, CCs do not show proton transfer. CC formation is an alternative approach which can be used to improve the bioavailability of APIs like EFV. Pharmaceutical CCs of EFV have been prepared with oxalic acid dihydrate 
and citric acid monohydrate CCs with 1,4-cyclohexanedione and 4,4 -bipyridine to name a few [4-6]. Although EFV exists as a total of 19 different solid forms: Forms I-V, H1, $\alpha, \beta, \mathrm{y}, \mathrm{y} 1, \mathrm{y} 2, \omega$, $\delta, \mathrm{N}, \mathrm{O}$ and $\mathrm{P}$ as well as three amorphous forms [7-11], there are few reports on the solid-state structures and behaviour of EFV CCs with few successes being reported, it was decided to utilize crystal engineering techniques to formulate new multicomponent molecular crystals of EFV [5]. Such knowledge may be useful to guide the design of other crystal forms of the drug with desired pharmaceutical characteristics, thereby improving its bioavailability, which is a major concern in pharmaceutical formulations.

The study focused on the co-crystallization of EFV with BZA (benzanilide) as coformer by means of the solvent evaporation technique. An array of methods can be used for the solid state characterisation of EFV co-crystals. FTIR (Fourier transform infrared) spectroscopy and X-ray Powder Diffraction (XRD) were chosen in this study. FTIR spectroscopy guides the identification of the CCs formed by indicating changes in frequencies, relative intensities, band contours and the number of bands. Differences in spectra give an interpretation to the internal arrangement of crystals whilst powder XRD can be utilised as a useful method to identify new phases. A different XRD pattern for the formed CCs compared to that of the individual components endorses the formation of a new phase $[4,12]$.

\section{Materials and Methods}

\subsection{Reagents}

Stocrin ${ }^{\circledR}$ tablets (600 mg EFV API) (Reg. No. 37/20.2.8/0628) was supplied by Shalom Laboratory Supplies C.C., Durban, South Africa. Methanol, acetonitrile and chloroform were of AR-grade and were purchased from Merck and used without further purification.

\subsection{Efavirenz API (Active Pharmaceutical Ingredient)}

\section{Extraction Method}

Stocrin tablets containing $600 \mathrm{mg}$ of EFV API was crushed with a mortar and pestle then dissolved in petroleum ether $(15 \mathrm{~mL})$ and filtered via gravity and left overnight in the fume hood to evaporate the solvent off.

\subsection{Preparation of Co-crystals}

The most effective and general method for preparing drug polymorphs is crystallization from solution. EFV (10 mg) was dissolved in petroleum ether $(5 \mathrm{~mL})$ and the solvent was evaporated off using a steam bath until half of the solution remained, which was then cooled for about 15 minutes and kept at $28{ }^{\circ} \mathrm{C}$ for recrystallization (slow recrystallization). The selection of temperature is based purely on the effects of temperature on the nucleation rate which has both thermodynamic and kinetic implications, principally in regards to enantiotropic polymorphs like those seen in EFV. The following morning crystals which were suitable for spectroscopic analysis were observed at the bottom of the vial. A 1:1 stoichiometric ratio of EFV (10.6 mg, $0.03358 \mathrm{mmol}$ ) and BZA (6 mg, 197.24 $\mathrm{g} / \mathrm{mol}$ ) was dissolved in a minimum amount of EtOH (5 $\mathrm{mL})$. The solution was then heated to $80{ }^{\circ} \mathrm{C}$ followed by crystallization by slow evaporation from the solvent over 7 days.

\section{Characterization of Co-crystals}

\subsection{FTIR (Fourier Transform Infrared) Spectroscopy}

The FTIR spectra of EFV, coformers, physical mixture \& their co-crystals were obtained on Spectrum RX I, FT-IR spectrometer (Perkin Elmer, UK) over the range 400-4,000 $\mathrm{cm}^{-1}$. Dry $\mathrm{KBr}$ (50 mg) was finely ground in mortar and samples (1-2 mg) were subsequently added and gently mixed in order to avoid trituration of the crystals so as to minimize scattering losses and absorption band distortions due to the hygroscopic nature of $\mathrm{KBr}$, as finely powdered potassium bromide readily absorbs humidity from the 
air leading to increased observed background in certain ranges. A manual press was used to form the pellets.

\subsection{X-ray Powder Diffraction (XRD)}

The material submitted was scanned to prepare for XRD analysis using a backloading preparation method. It was analysed with a PANalytical Empyrean diffractometer with PIXcel detector and fixed slits with Fe filtered Co-K $\alpha$ radiation. The phases were identified using X'Pert Highscore plus software. The conditions set were, voltage at $40 \mathrm{kV}$, current at $30 \mathrm{~mA}$, scanning speed at $2 \%$ min while the temperature of acquisition was at room temperature.

\section{Results and Discussion}

\subsection{FTIR (Fourier Transform Infrared) Spectroscopy}

FTIR spectroscopy has become the principal method for the characterization and determination of molecular properties of solid-state forms. It can be used to monitor bonds and bound atoms, in addition to disturbances in vibrations. It is easy to determine the exact wave number $\left(\mathrm{cm}^{-1}\right)$ of the absorption bands, which are presented in the form of a spectral image with it.

Because polymorphs of other compounds might exhibit identical spectra in solution, solid samples of EFV were used in this study. The spectral analysis was carried out in two parts: firstly to identify the drug API by using characteristic bands, and secondly, to identify the new co-crystal formed. EFV is composed of a benzoxazinone framework with functional groups, trifluoromethyl and cyclopropylacetylene attached at C-4 (Fig. 1). From the structure of EFV, the characteristic bands in the fingerprint region are observed and presented in Table 1. FTIR is an excellent technique which gives insight into the kind of interactions occurring between API and coformer. The FTIR data of EFV and BZA and their respective physical mixtures are presented in Table 2 and Fig. 1. A perusal of Table 2 summarises the FTIR spectra of the polymoph obtained on crystallization with petroleum ether, and indicates that polymorph II formed and these values were in agreement with existing literature provided by Fandaruff et al. (2014) in Table 1.

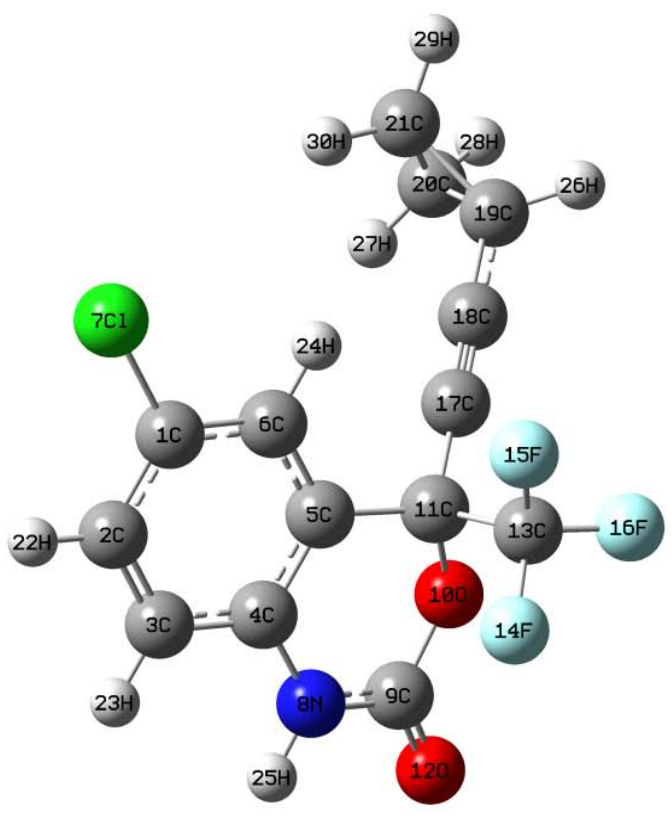

Fig. 1 Chemical structure of EFV [1]. 
Table 1 Characteristic infrared absorption bands of EFV [9].

\begin{tabular}{ll}
\hline Frequency $\left(\mathrm{cm}^{-1}\right)$ & Vibrational assignments \\
\hline 2,260 & Typical exocyclic triple bond stretching \\
1,757 & $\mathrm{C}=\mathrm{O}$ stretching \\
1,602 & Tertiary amide \\
$900-650$ & Aromatic ring \\
$1,350-1,120$ & $\mathrm{CF}_{3}$ \\
$1,096-1,089$ & $\mathrm{C}-\mathrm{Cl}$ stretching \\
3,180 & $\mathrm{~N}-\mathrm{H}$ stretching vibration \\
\hline
\end{tabular}

Table 2 The FTIR data of EFV and BZA and the EFV-BZA physical mixture.

\begin{tabular}{llll}
\hline IR frequencies & EFV & BZA & EFV-BZA \\
\hline N-H stretch & $3,312.39$ & $3,343.58$ & $3,299.71$ \\
$\mathrm{C}=\mathrm{O}$ & $1,749.7$ & $1,654.34$ & $1,739.87$ \\
$\mathrm{C} \equiv \mathrm{C}$ & $2,249.90$ & $*$ & $2,249.18$ \\
$\mathrm{C}-\mathrm{F}$ & $1,315.88,1,240.97$ & $*$ & $1,320.34,1,240.55$ \\
$\mathrm{C}-\mathrm{Cl}$ & $1,036.10$ & $*$ & $1,028.09$ \\
\hline
\end{tabular}

* N/A: Not applicable as functionalities are absent within the molecule.

The FTIR spectrum of EFV, Fig. 2, shows a strong $\mathrm{N}-\mathrm{H}$ stretch and $\mathrm{C}=\mathrm{O}$ stretch of the amide group at 3,312.39 and 1,749.7 $\mathrm{cm}^{-1}$ respectively, the C-C stretch is due to an alkyne $(\mathrm{C} \equiv \mathrm{C})$ at $2,249.90 \mathrm{~cm}^{-1}$, the $\mathrm{C}$-F of $-\mathrm{CF}_{3}$ is observed at $1,315.88,1,240.97 \mathrm{~cm}^{-1}$ and the C-Cl stretch appears at $1,036.10 \mathrm{~cm}^{-1}$.

FTIR spectra of EFV-BZA, Fig. 2, however, shows significant changes in the FTIR frequencies in prepared CCs when compared to the pure drug thereby indicating that hydrogen bonding had occurred in the CCs. Firstly the N-H stretch of the amide group of EFV has shifted from $3,312.39 \mathrm{~cm}^{-1}$ to $3,299.71 \mathrm{~cm}^{-1}$ while the $\mathrm{C}=\mathrm{O}$ stretch has shifted from $1,749.70 \mathrm{~cm}^{-1}$ to $1,739.87 \mathrm{~cm}^{-1}$ suggesting that both $\mathrm{N}-\mathrm{H}$ and $\mathrm{C}=\mathrm{O}$ of $\mathrm{EFV}$ and the $\mathrm{N}-\mathrm{H}$ and $\mathrm{C}=\mathrm{O}$ of group of BZA are participating in hydrogen bonding in the EFV-BZA crystalline complex. Furthermore, from the FTIR data it is seen that the N-H is shifted to a higher wave number. This illustrates the formation of hydrogen bonds between the NH of EFV with the BZA N(hydrogen bonding occurs between the NH---N and $\mathrm{NH}---\mathrm{O}=\mathrm{C}$ ) (Fig. 3), signifying the formation of a new phase. Campos de Melo et al., observed common hetero-and homosynthons of EFV proposed in Fig. 3 [5]. This observation also relates with that of Jordaan et al., who discovered that EFV undergoes inter- and intra-molecular hydrogen bonding as the oxazinan-2-one substrate is composed of an electron donor group (amino) and an electron acceptor group (carbonyl) allowing interactions with the BZA coformer [1, 13]. As a result of the formation of hydrogen bonds, the $\mathrm{NH}$ bond becomes increasingly rigid and relatively shorter so that the stretch frequency increases. These hydrogen bonding interactions should render the EFV-BZA crystalline complex more soluble in aqueous media.

\subsection{X-ray Powder Diffraction (XRD)}

X-ray Powder Diffraction (XRD) is a useful method for fast identification of the new phases. A different XRD pattern for the CCs from those of the individual components confirms the formation of a new phase. In a diffraction pattern, the strongest peak is allotted an intensity value of 100 , the rest of the peaks are thus scaled relative to that value. Based on this the XRD data for EFV, BZA, and their respective co-crystal is presented in Table 3 at their maximum intensity and the XRD patterns have been depicted in Figs. 4 and 5. The XRD pattern of EFV (Appendix 1) shows some characteristic peaks that are in line with that in literature at approximately $7.1006^{\circ}, 13.6286^{\circ}$, $24.6982^{\circ}, 15.374^{\circ}, 16.491^{\circ}$ and $31.754^{\circ}$ while the XRD 

of the Anti-HIV Drug Efavirenz

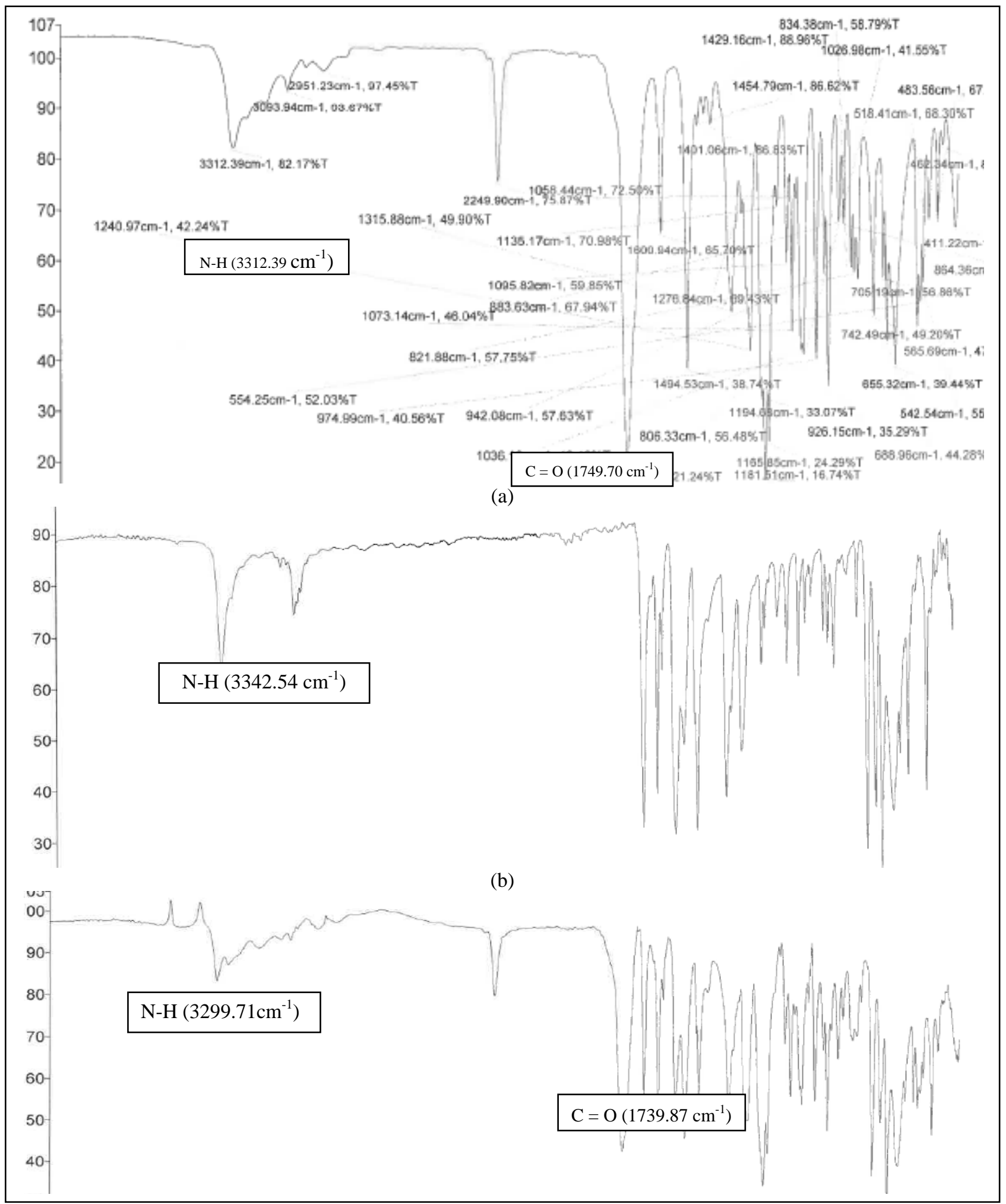

Fig. 2 FTIR spectra of (a) EFV (b) BZA (c) EFV-BZA physical mixture. 

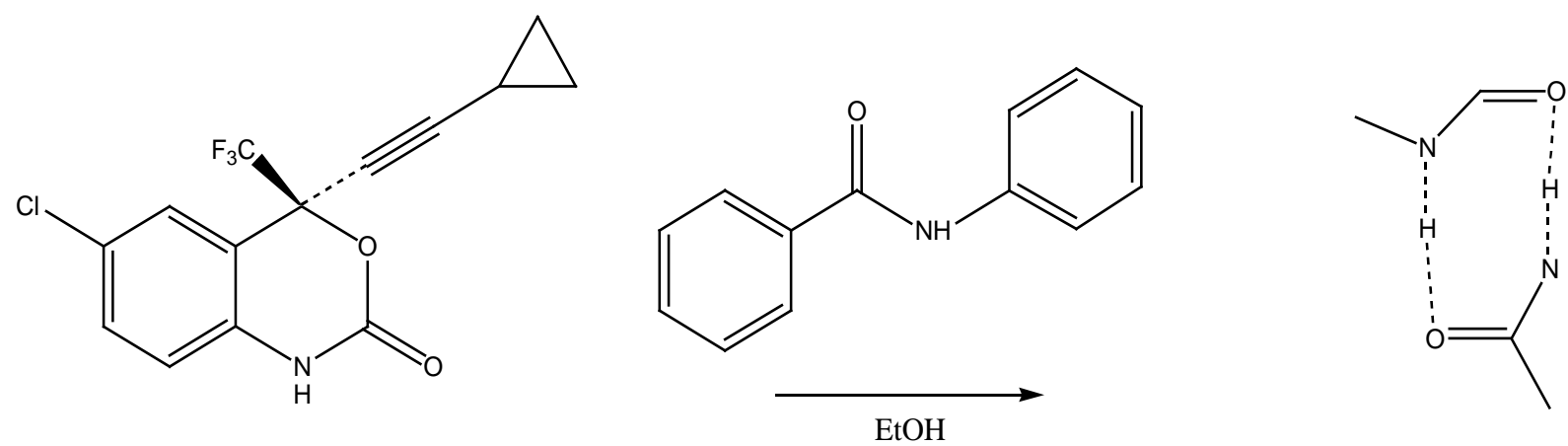

(I)
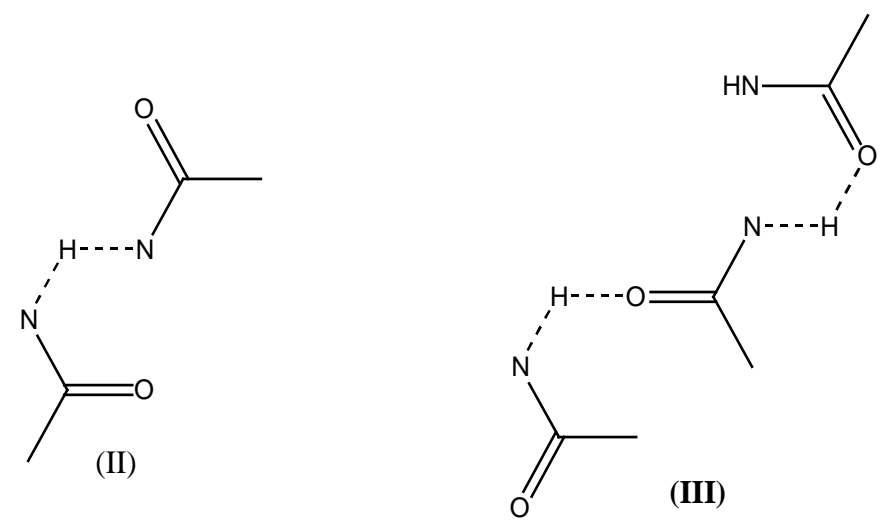

Fig. 3 EFV and BZA with proposed hetero- and homo-synthons I, II and III [5].

Table 3 XRD data of EFV and BZA and the EFV-BZA physical mixture.

\begin{tabular}{lll}
\hline & $*$ Pos. $\left[{ }^{\circ} 2 \theta\right]$ & Rel. Int. [\%] \\
\hline EFV & 31.9709 & 100 \\
BZA & 27.1126 & 100 \\
EFV-BZA & 30.8005 & 100 \\
\hline
\end{tabular}

* The diffraction peak position is recorded as the angle 2 theta $(2 \theta)$.

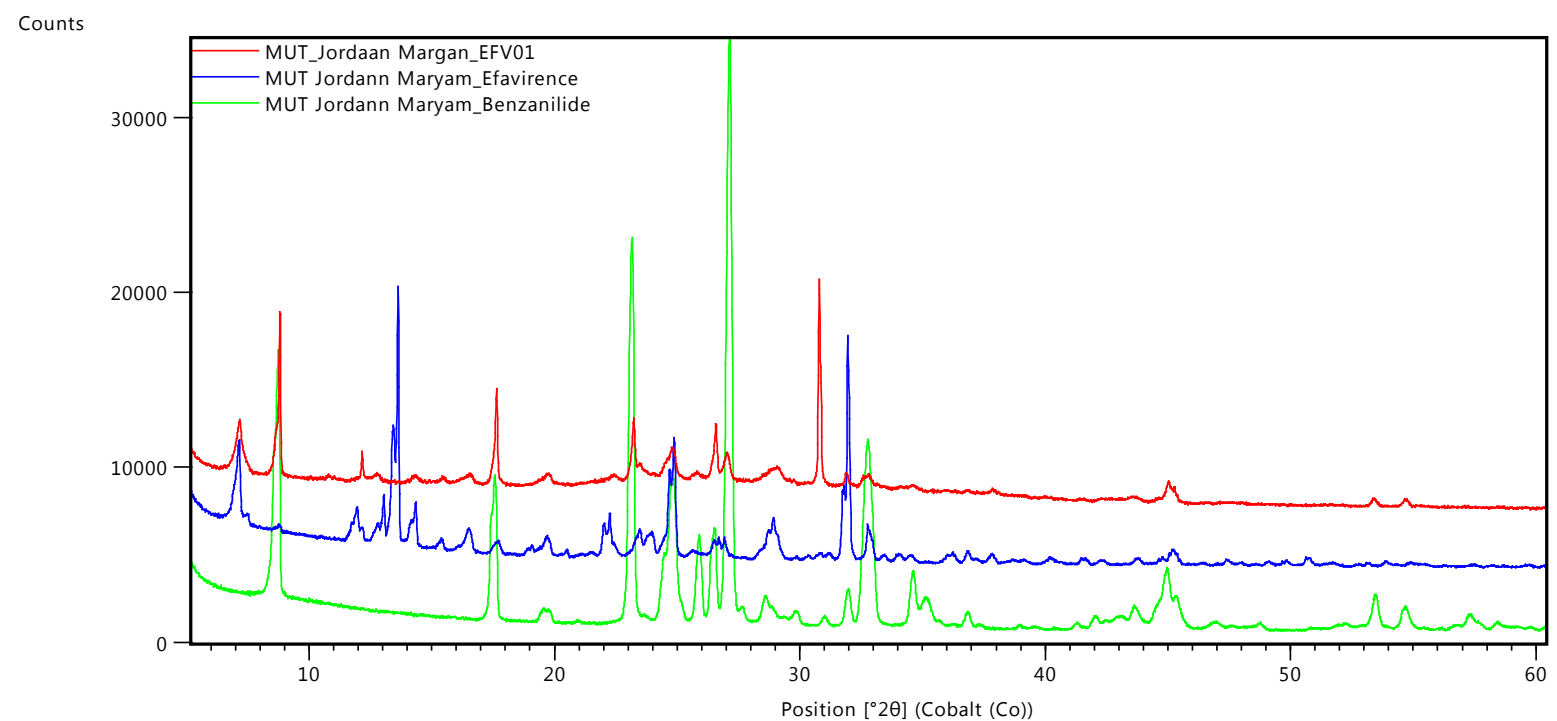

Fig. 4 Overlap of 3 XRD patterns of EFV-BZA co-crystal (red), EFV (blue) and BZA (green). 

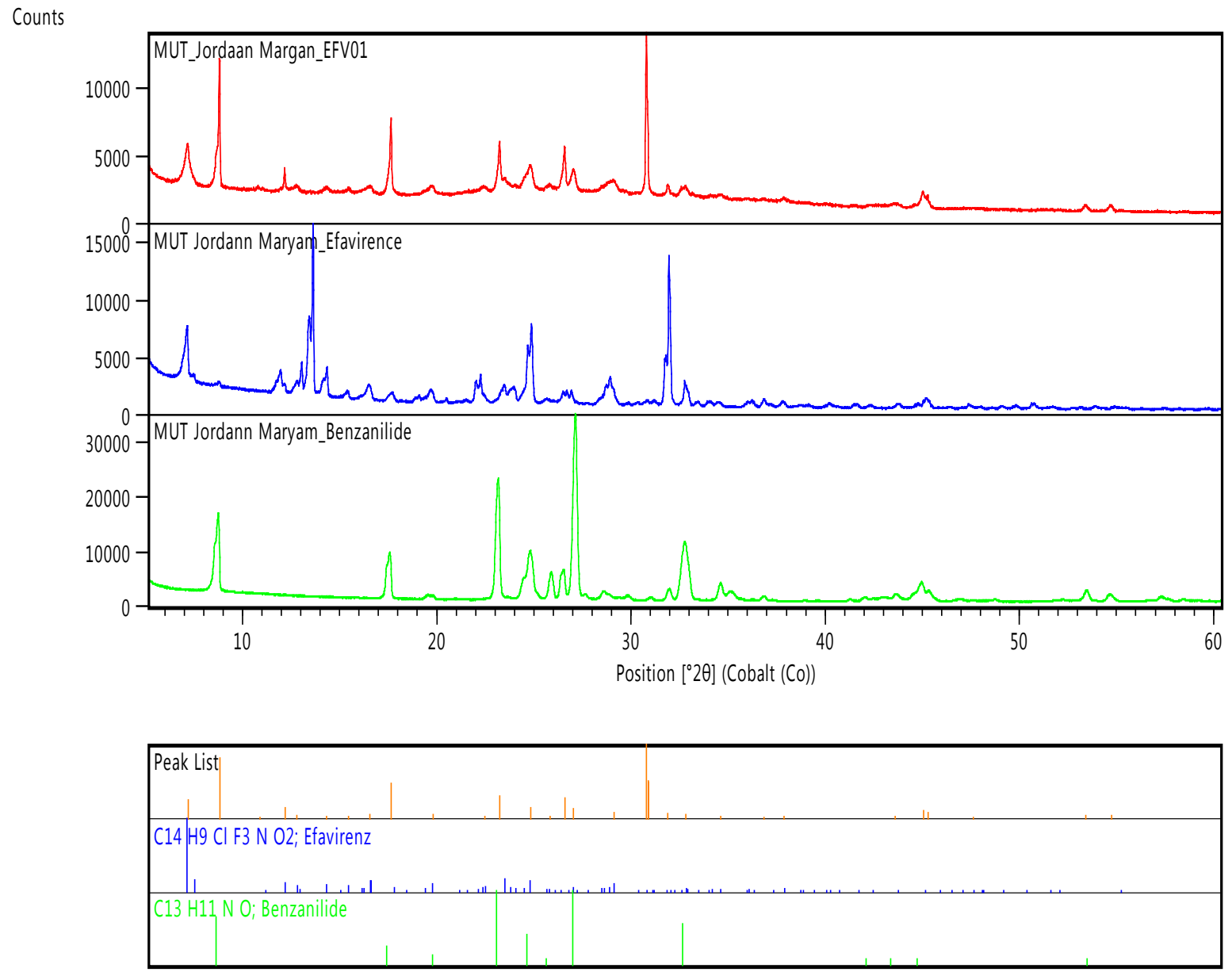

Fig. 5 XRD patterns of EFV-BZA co-crystal (red), EFV (blue) and BZA (green).

pattern of BZA (Appendix 2) shows characteristic peaks at approximately $8.593^{\circ}, 8.751^{\circ}, 17.433^{\circ}$, $17.587^{\circ}, 23.130^{\circ}, 24.782^{\circ}$ and $32.742^{\circ}$. However, in the XRD pattern of EFV-BZA (Appendix 3), one unique peak which is absent in both EFV and BZA has appeared at $30.8005^{\circ}$ (100\% intensity) suggesting the formation of a new solid phase (Table 3).The differences seen in Table 3 are most likely attributed to the hydrogen bonding interactions between EFV and BZA it is therefore obvious that there has been a transformation in the crystalline lattices of EFV and the BZA coformer and a new phase has been formed.

\section{Conclusions}

In our pursuit to improve the solubility of EFV, co-crystallization of EFV with BZA as coformer was investigated by means of the solvent evaporation method. The FTIR data indicate the formation of a novel CC as evidenced by significant changes in the FTIR frequencies in prepared CCs when compared to the pure drug, which indicated that hydrogen bonding had occurred within the CCs. Furthermore the XRD pattern of EFV and BZA shows characteristic peaks, however, in the XRD pattern of EFV-BZA, one unique peak which is absent in both EFV and BZA has appeared at $30.8005^{\circ}$ (100\% intensity) suggesting the formation of a new solid phase. The research is significant in understanding the complex chemistry of EFV based CC formulation. Further research can thus be directed at first evaluating the physicochemical properties and improved bioavailability of the EFV-BZA CC. Secondly based on information from literature, successful pharmaceutical CCs of EFV were centred on amide-, carboxylic acid- and pyridine-type 

of the Anti-HIV Drug Efavirenz

coformers with sufficient hydrogen bond donor and acceptor groups fortifying our selection of BZA as coformer. In light of this data further CC formulations can be prepared with coformers comprising a water soluble BZA-type scaffold with GRAS (Generally Recognized as Safe) status as BZA in its pure form is not GRAS approved.

\section{Funding}

This work was supported by the National Research foundation [grant number (NRF grant UID: 102425)] and MUT (Mangosuthu University of Technology). We would like to thank Dr. Sabine Verryn from the XRD Analytical and Consulting CC for the XRD analysis.

\section{References}

[1] Jordaan, M. A., Singh, P., and Martincigh, B. S. 2016. “A Combined TD-DFT and Spectroscopic Investigation of the Solute-Solvent Interactions of Efavirenz.” Spectrochim. Acta Mol. Biomol. Spectrosc. 157: 204-10.

[2] Lahamage, S. R., et al. 2016. "Pharmaceutical Co-Crystallization.” Asian J. Res. Pharm. Sci. 6 (1): 51-8.

[3] Yamamotoa, K., Tsutsumi, S., and Ikeda, Y. 2012. "Establishment of Cocrystal Cocktail Grinding Method for Rational Screening of Pharmaceutical Cocrystals.” Int. J. Pharm. 437: 162-71.

[4] Chadha, R., Anupam, S., Poonam, A., Somnath, C., and Dharamvirsingh, J. 2012. "Cocrystals of Efavirenz with Selected Coformers: Preparation and Characterization.” $J$ Pharm. Pharm. Sci. 15 (2): 234-51.

[5] Campos de Melo, A. R., de Amorim, I. F., de Lima
Cirqueira, M., and Martins, F. T. 2013. "Toward Novel Solid-State Forms of the Anti-HIV Drug Efavirenz: From Low Screening Success to Cocrystals Engineering Strategiesand Discovery of a New Polymorph.” Cryst. Growth Des. 13: 1558-69.

[6] Mahapatra, S., Thakur, T. S., Joseph, S., Varughese, S., and Desiraju, G. R. 2010. "New Solid State Forms of the Anti-HIV Drug Efavirenz. Conformational Flexibility and High Z'Issues.” Cryst. Growth Des. 10: 3191-202.

[7] Reddy, B. P., et al. 2006. Novel Polymorphs of Efavirenz. Patent: US 2006/0235008 A1.

[8] Wardhana, Y. W. 2017. “Observation of Polymorphic Transformation of Amorphous Efavirenz during Heating and Grinding Processes Using Raman Spectroscopy.” Res. J. Pharm., Biol. Chem. Sci. 8 (1S): 280-6.

[9] Fandaruff, C., Rauber, G. S., Araya-Sibaja, A. M., Pereira, R. N., Campos, C. E. M. D., Rocha, H. V. A., et al. 2014. "Polymorphism of Anti-HIV Drug Efavirenz: Investigations on Thermodynamic and Dissolution Properties.” J. Excipients and Food Chem. 5 (3): 152-60.

[10] Clarke, W., and Kukura, J. L. 1998. Process for the Crystallisation of a Reverse Transcriptase Inhibitor Using an Anti-solvent. Patent: US 5,965,729.

[11] Radesca, L., et al. 1998. Crystalline Efavirenz. Patent: WO 99/64405.

[12] Mahapatra, A. K., and Murthy, P. N. 2014. "Solubility and Dissolution Rate Enhancement of Efavirenz by Inclusion Complexation and Liquid Anti-solvent Precipitation Technique.” J. Chem. Pharm. Res. 6 (4): 1099-106.

[13] Jordaan, M. A., and Shapi, M. M. 2017. "Investigation of the Solvent-Dependent Photolysis of a Nonnucleoside Reverse-Transcriptase Inhibitor (NNRTI), Antiviral Agent Efavirenz.” Antivir. Chem. Chemother. 2017: 1-11. DOI: 10.1177/2040206617730170. 

of the Anti-HIV Drug Efavirenz

Appendix 1 XRPD data of EFV.

\begin{tabular}{|c|c|c|c|c|c|}
\hline No. & Pos. $\left[{ }^{\circ} 2 \theta\right]$ & D-spacing [A] & Backgr. [cts] & Height [cts] & Rel. Int. [\%] \\
\hline 1 & 7.1006(8) & 14.44498 & 3,629.35 & $2,778.87$ & 23.79 \\
\hline 2 & $7.504(6)$ & 13.6695 & $3,446.77$ & 153.17 & 1.31 \\
\hline 3 & $8.777(5)$ & 11.68944 & $3,054.42$ & 254.59 & 2.18 \\
\hline 4 & $11.929(1)$ & 8.60856 & $2,546.78$ & $1,320.45$ & 11.3 \\
\hline 5 & $12.181(5)$ & 8.43074 & $2,524.96$ & 160.43 & 1.37 \\
\hline 6 & $12.796(4)$ & 8.02689 & $2,451.65$ & 550.51 & 4.71 \\
\hline 7 & 13.0436(8) & 7.87547 & 2,416.39 & $1,766.75$ & 15.13 \\
\hline 8 & 13.4473(8) & 7.64008 & $2,352.48$ & $4,407.86$ & 37.74 \\
\hline 9 & 13.6286(2) & 7.5389 & 2,322.16 & $9,728.02$ & 83.28 \\
\hline 10 & $14.174(4)$ & 7.25011 & $2,230.38$ & 898.73 & 7.69 \\
\hline 11 & $14.3450(9)$ & 7.16423 & 2,197.62 & $1,540.24$ & 13.19 \\
\hline 12 & 15.374(3) & 6.68744 & $2,056.36$ & 355.04 & 3.04 \\
\hline 13 & $16.491(2)$ & 6.23702 & $1,912.11$ & 876.6 & 7.5 \\
\hline 14 & 17.654(3) & 5.82911 & $1,779.32$ & 456.76 & 3.91 \\
\hline 15 & 19.084(3) & 5.39617 & $1,705.73$ & 377.75 & 3.23 \\
\hline 16 & 19.681(2) & 5.23402 & $1,697.89$ & 691.34 & 5.92 \\
\hline 17 & 20.492(3) & 5.02876 & 1,671 & 218.74 & 1.87 \\
\hline 18 & 21.491(7) & 4.79771 & $1,668.78$ & 125.74 & 1.08 \\
\hline 19 & 22.021(1) & 4.68354 & $1,681.96$ & $1,080.42$ & 9.25 \\
\hline 20 & $22.252(1)$ & 4.63562 & 1,687.69 & 1,638.39 & 14.03 \\
\hline 21 & $23.447(2)$ & 4.40226 & $1,701.76$ & $1,014.35$ & 8.68 \\
\hline 22 & $23.900(2)$ & 4.32006 & $1,695.61$ & 801.27 & 6.86 \\
\hline 23 & 24.6982(9) & 4.18252 & $1,671.5$ & 3,733.78 & 31.97 \\
\hline 24 & $24.8778(5)$ & 4.1528 & $1,662.75$ & $3,971.21$ & 34 \\
\hline 25 & 25.687(7) & 4.02413 & $1,642.64$ & 164.34 & 1.41 \\
\hline 26 & $26.508(4)$ & 3.90158 & $1,607.02$ & 578.04 & 4.95 \\
\hline 27 & $26.710(2)$ & 3.87256 & $1,595.27$ & 615.06 & 5.27 \\
\hline 28 & $26.939(2)$ & 3.84032 & $1,582.01$ & 748.87 & 6.41 \\
\hline 29 & $28.704(4)$ & 3.60866 & $1,478.54$ & 916.31 & 7.84 \\
\hline 30 & 28.922(3) & 3.58205 & $1,474.33$ & 1,332.24 & 11.41 \\
\hline 31 & 29.107(6) & 3.55971 & 1,469.95 & 414.34 & 3.55 \\
\hline 32 & 29.862(8) & 3.47168 & 1,438.99 & 106.46 & 0.91 \\
\hline 33 & $30.322(9)$ & 3.42026 & $1,445.11$ & 144.93 & 1.24 \\
\hline 34 & $30.817(8)$ & 3.36662 & $1,467.89$ & 217.48 & 1.86 \\
\hline 35 & $31.181(7)$ & 3.32823 & $1,481.25$ & 195.25 & 1.67 \\
\hline 36 & $31.754(2)$ & 3.26976 & $1,490.41$ & 2,994.27 & 25.63 \\
\hline 37 & $31.838(4)$ & 3.26127 & $1,490.71$ & 1,619.97 & 13.87 \\
\hline 38 & $31.9709(4)$ & 3.2481 & $1,490.7$ & $11,680.46$ & 100 \\
\hline 39 & $32.776(2)$ & 3.17046 & $1,475.82$ & $1,547.53$ & 13.25 \\
\hline 40 & $32.927(4)$ & 3.15627 & $1,472.67$ & 755.66 & 6.47 \\
\hline 41 & $33.420(5)$ & 3.11104 & $1,452.1$ & 178.7 & 1.53 \\
\hline 42 & $34.054(4)$ & 3.05476 & $1,408.48$ & 229.13 & 1.96 \\
\hline 43 & $34.509(5)$ & 3.01568 & $1,366.54$ & 234.62 & 2.01 \\
\hline 44 & $36.02(1)$ & 2.8928 & 1,326.23 & 240.32 & 2.06 \\
\hline 45 & $36.257(8)$ & 2.87487 & 1,330.41 & 283.6 & 2.43 \\
\hline 46 & $36.855(2)$ & 2.82976 & 1,321.18 & 462.73 & 3.96 \\
\hline 47 & $37.815(3)$ & 2.7605 & 1,284.32 & 321.92 & 2.76 \\
\hline
\end{tabular}


(Appendix 1 continued)

\begin{tabular}{|c|c|c|c|c|c|}
\hline No. & Pos. $\left[{ }^{\circ} 2 \theta\right]$ & D-spacing [A] & Backgr. [cts] & Height [cts] & Rel. Int. [\%] \\
\hline 48 & $38.70(2)$ & 2.69959 & $1,244.45$ & 112.32 & 0.96 \\
\hline 49 & 39.13(1) & 2.67108 & $1,221.13$ & 127.09 & 1.09 \\
\hline 50 & $40.215(4)$ & 2.60194 & 1,193.81 & 297 & 2.54 \\
\hline 51 & $41.489(5)$ & 2.52542 & 1,192.26 & 240.93 & 2.06 \\
\hline 52 & $41.674(4)$ & 2.51469 & $1,192.3$ & 270.77 & 2.32 \\
\hline 53 & $42.300(6)$ & 2.47916 & $1,184.33$ & 150.86 & 1.29 \\
\hline 54 & $43.744(4)$ & 2.40112 & 1,198.95 & 223.18 & 1.91 \\
\hline 55 & 44.781(4) & 2.34829 & $1,208.76$ & 298.38 & 2.55 \\
\hline 56 & $45.079(2)$ & 2.33357 & $1,205.11$ & 363.75 & 3.11 \\
\hline 57 & $45.244(9)$ & 2.3255 & $1,203.09$ & 506.34 & 4.33 \\
\hline 58 & $46.41(1)$ & 2.27019 & $1,153.74$ & 71.49 & 0.61 \\
\hline 59 & $47.415(4)$ & 2.22477 & 1,118.35 & 259.12 & 2.22 \\
\hline 60 & 47.971(7) & 2.20049 & $1,107.41$ & 122.93 & 1.05 \\
\hline 61 & 49.095(6) & 2.15311 & $1,124.27$ & 164.95 & 1.41 \\
\hline 62 & 49.797(5) & 2.12465 & $1,136.55$ & 167.92 & 1.44 \\
\hline 63 & $50.645(3)$ & 2.09135 & $1,129.44$ & 378.82 & 3.24 \\
\hline 64 & $50.805(5)$ & 2.0852 & $1,126.54$ & 259.45 & 2.22 \\
\hline 65 & $51.65(1)$ & 2.05324 & $1,107.44$ & 100.69 & 0.86 \\
\hline 66 & $53.123(4)$ & 2.00041 & $1,097.52$ & 163.18 & 1.4 \\
\hline 67 & $53.890(5)$ & 1.97404 & $1,092.73$ & 175.65 & 1.5 \\
\hline 68 & 54.95(1) & 1.93872 & $1,084.61$ & 125.4 & 1.07 \\
\hline 69 & 57.4712 & 1.86057 & $1,041.01$ & 111.34 & 0.95 \\
\hline 70 & 58.2626 & 1.83747 & $1,040.94$ & 75.65 & 0.65 \\
\hline 71 & 59.6792 & 1.79773 & $1,047.62$ & 67.69 & 0.58 \\
\hline 72 & 60.4539 & 1.77683 & $1,042.56$ & 62.5 & 0.54 \\
\hline 73 & 62.9804 & 1.71245 & $1,008.83$ & 62.87 & 0.54 \\
\hline 74 & 63.5254 & 1.69928 & $1,009.03$ & 50.37 & 0.43 \\
\hline 75 & 64.3752 & 1.67921 & $1,002.25$ & 95.17 & 0.81 \\
\hline 76 & 64.8779 & 1.66761 & 995.29 & 400.87 & 3.43 \\
\hline 77 & 65.0412 & 1.66388 & 992.48 & 272.19 & 2.33 \\
\hline 78 & 66.3741 & 1.63417 & 971.66 & 87.25 & 0.75 \\
\hline 79 & 68.7977 & 1.58333 & 946.2 & 23.99 & 0.21 \\
\hline 80 & 70.0222 & 1.55909 & 934.62 & 94.82 & 0.81 \\
\hline 81 & 70.9857 & 1.54065 & 916.75 & 85.85 & 0.73 \\
\hline 82 & 73.2588 & 1.49925 & 886.88 & 170.62 & 1.46 \\
\hline
\end{tabular}



of the Anti-HIV Drug Efavirenz

Appendix 2 XRPD data of BZA.

\begin{tabular}{|c|c|c|c|c|c|}
\hline No. & Pos. $\left[{ }^{\circ} 2 \theta\right]$ & D-spacing $[\AA]$ & Backgr. [cts] & Height [cts] & Rel. Int. [\%] \\
\hline 1 & $8.593(1)$ & 11.9398 & $2,681.01$ & $5,775.07$ & 24.94 \\
\hline 2 & $8.7513(6)$ & 11.7242 & $2,648.8$ & $8,538.53$ & 36.88 \\
\hline 3 & $17.433(2)$ & 5.90269 & 1,323.97 & 3,958.62 & 17.1 \\
\hline 4 & $17.587(1)$ & 5.85134 & 1,313.66 & $5,313.78$ & 22.95 \\
\hline 5 & 19.541(7) & 5.27095 & $1,151.34$ & 503.82 & 2.18 \\
\hline 6 & 19.793(7) & 5.20458 & $1,146.47$ & 407.2 & 1.76 \\
\hline 7 & $20.96(2)$ & 4.91808 & $1,089.16$ & 40.07 & 0.17 \\
\hline 8 & 23.1304(2) & 4.46175 & $1,312.22$ & $1,5412.25$ & 66.57 \\
\hline 9 & $24.461(6)$ & 4.22246 & $1,509.61$ & 2092.9 & 9.04 \\
\hline 10 & $24.782(5)$ & 4.16862 & $1,539.8$ & 4,926.39 & 21.28 \\
\hline 11 & 24.940(3) & 4.14254 & $1,547.74$ & $2,123.88$ & 9.17 \\
\hline 12 & $25.8(1)$ & 4.00063 & $1,547.11$ & $1,992.66$ & 8.61 \\
\hline 13 & $25.94(6)$ & 3.9851 & $1,539.21$ & $1,641.39$ & 7.09 \\
\hline 14 & 26.399(3) & 3.9174 & $1,494.41$ & $2,624.26$ & 11.33 \\
\hline 15 & $26.542(2)$ & 3.8966 & $1,477.14$ & $3,600.24$ & 15.55 \\
\hline 16 & 27.1126(1) & 3.81614 & $1,390.67$ & $23,152.01$ & 100 \\
\hline 17 & $27.630(4)$ & 3.746 & $1,307.65$ & 433.29 & 1.87 \\
\hline 18 & $28.646(2)$ & 3.61583 & $1,202.35$ & $1,174.19$ & 5.07 \\
\hline 19 & 29.816(3) & 3.4769 & 1,079.9 & 500.18 & 2.16 \\
\hline 20 & 31.004(3) & 3.34682 & 983.29 & 325.96 & 1.41 \\
\hline 21 & 31.957(1) & 3.24949 & $1,042.3$ & $1,397.12$ & 6.03 \\
\hline 22 & $32.742(4)$ & 3.17359 & $1,055.98$ & $6,881.4$ & 29.72 \\
\hline 23 & $32.975(6)$ & 3.15183 & $1,052.82$ & $1,555.86$ & 6.72 \\
\hline 24 & $34.5(2)$ & 3.01284 & $1,063.35$ & 997.98 & 4.31 \\
\hline 25 & $34.6(1)$ & 3.00447 & $1,066.44$ & $1,406.93$ & 6.08 \\
\hline 26 & $35.128(5)$ & 2.96415 & $1,081.59$ & 967.22 & 4.18 \\
\hline 27 & 37(154214263084576) & 2.85167 & 996.25 & 0 & 0 \\
\hline 28 & $36.836(2)$ & 2.83115 & 962.4 & 557.25 & 2.41 \\
\hline 29 & 37.33(1) & 2.79496 & 896.6 & 83.28 & 0.36 \\
\hline 30 & 38.961(7) & 2.68228 & 786.43 & 145.56 & 0.63 \\
\hline 31 & $39.60(2)$ & 2.64098 & 767.95 & 73.7 & 0.32 \\
\hline 32 & $41.270(5)$ & 2.53824 & 836.83 & 176.52 & 0.76 \\
\hline 33 & 42.031(3) & 2.49429 & 983.37 & 348.35 & 1.5 \\
\hline 34 & $43.00(1)$ & 2.44062 & $1,079.98$ & 263.66 & 1.14 \\
\hline 35 & 43.657(3) & 2.40566 & $1,117.61$ & 609.93 & 2.63 \\
\hline 36 & 44.561(9) & 2.35931 & $1,095.79$ & 559.69 & 2.42 \\
\hline 37 & $44.936(2)$ & 2.34062 & $1,061.28$ & $2,234.36$ & 9.65 \\
\hline 38 & $45.363(7)$ & 2.31972 & 996.68 & 900.85 & 3.89 \\
\hline 39 & $46.900(7)$ & 2.24778 & 837.05 & 211.47 & 0.91 \\
\hline 40 & $47.60(4)$ & 2.21667 & 866.16 & 64.11 & 0.28 \\
\hline 41 & $48.713(5)$ & 2.16897 & 794.28 & 209.6 & 0.91 \\
\hline 42 & $50.96(2)$ & 2.07941 & 765.32 & 48.68 & 0.21 \\
\hline 43 & $52.11(2)$ & 2.03643 & 861.92 & 122.83 & 0.53 \\
\hline 44 & $53.433(1)$ & 1.98966 & 894.56 & $1,395.77$ & 6.03 \\
\hline 45 & $54.59(5)$ & 1.95049 & 836.14 & 529.22 & 2.29 \\
\hline 46 & $54.7(2)$ & 1.94589 & 830.83 & 494.48 & 2.14 \\
\hline 47 & $56.72(3)$ & 1.88316 & 802.69 & 145.42 & 0.63 \\
\hline
\end{tabular}


(Appendix 2 continued)

\begin{tabular}{llllll}
\hline No. & Pos. $\left[{ }^{\circ} 2 \theta\right]$ & D-spacing $[\AA]$ & Backgr. [cts] & Height [cts] & Rel. Int. [\%] \\
\hline 48 & $57.324(3)$ & 1.86493 & 851.22 & 487.29 & 2.1 \\
49 & $58.427(6)$ & 1.83275 & 880.41 & 211.59 & 0.91 \\
50 & $60(65847309202276352)$ & 1.79769 & 843.51 & 0 & 0 \\
51 & $60.54(2)$ & 1.7745 & 797.84 & 147.96 & 0.64 \\
52 & $61.36(1)$ & 1.75312 & 774.38 & 73.44 & 0.32 \\
53 & $63.361(8)$ & 1.70324 & 643.36 & 109.43 & 0.47 \\
54 & $64.783(9)$ & 1.66977 & 635.74 & 111.9 & 0.48 \\
55 & $65.53(1)$ & 1.6528 & 618.41 & 105.6 & 0.46 \\
56 & $66.91(2)$ & 1.62257 & 596 & 54.71 & 0.24 \\
57 & $67.99(7)$ & 1.59995 & 604.77 & 30.63 & 0.13 \\
58 & $70.034(9)$ & 1.55885 & 551.96 & 67.16 & 0.29 \\
59 & 70.8516 & 1.54319 & 546.22 & 79.94 & 0.35 \\
60 & 73.1666 & 1.50087 & 494.84 & 93.39 & 0.4 \\
61 & 73.9888 & 1.48654 & 497.23 & 77.47 & 0.33
\end{tabular}

Appendix 3 XRPD Data of EFV-BZA CC.

\begin{tabular}{|c|c|c|c|c|c|}
\hline No. & Pos. $\left[{ }^{\circ} 2 \theta\right]$ & D-spacing [A] & Backgr. [cts] & Height [cts] & Rel. Int. [\%] \\
\hline 1 & 7.161(1) & 14.32425 & $2,822.35$ & $1,975.55$ & 18 \\
\hline 2 & $8.7978(3)$ & 11.66236 & $2,515.73$ & $5,464.61$ & 49.79 \\
\hline 3 & $10.82(1)$ & 9.49142 & 2,318 & 125.58 & 1.14 \\
\hline 4 & $12.1710(9)$ & 8.43775 & 2,253.93 & $1,240.15$ & 11.3 \\
\hline 5 & $12.725(8)$ & 8.07155 & $2,234.7$ & 218.19 & 1.99 \\
\hline 6 & $14.318(7)$ & 7.17788 & $2,190.81$ & 191.58 & 1.75 \\
\hline 7 & $15.454(7)$ & 6.65293 & $2,148.85$ & 174.02 & 1.59 \\
\hline 8 & $16.495(6)$ & 6.23563 & $2,078.08$ & 331 & 3.02 \\
\hline 9 & $17.6276(4)$ & 5.83788 & 1,996.69 & $3,771.51$ & 34.37 \\
\hline 10 & $19.687(5)$ & 5.23233 & $2,006.28$ & 385.13 & 3.51 \\
\hline 11 & $22.34(1)$ & 4.6169 & $2,231.02$ & 165.95 & 1.51 \\
\hline 12 & 23.2272(8) & 4.44342 & 2,312.16 & 2,313.08 & 21.08 \\
\hline 13 & $24.742(2)$ & 4.17527 & $2,374.46$ & $1,090.95$ & 9.94 \\
\hline 14 & $25.80(1)$ & 4.0061 & $2,365.68$ & 137.7 & 1.25 \\
\hline 15 & 26.5631(8) & 3.89362 & 2,335.14 & 2,208.95 & 20.13 \\
\hline 16 & $27.029(2)$ & 3.82773 & $2,302.76$ & 953.51 & 8.69 \\
\hline 17 & 28.958(5) & 3.5777 & $2,142.52$ & 564.24 & 5.14 \\
\hline 18 & $30.8005(2)$ & 3.36837 & 1,992.89 & $10,974.86$ & 100 \\
\hline 19 & $31.898(2)$ & 3.25531 & $1,922.49$ & 630.72 & 5.75 \\
\hline 20 & $32.746(4)$ & 3.17323 & $1,884.61$ & 452.23 & 4.12 \\
\hline 21 & $34.59(1)$ & 3.00924 & 1,739.93 & 159.68 & 1.45 \\
\hline 22 & 36.83(1) & 2.83171 & $1,562.3$ & 120.98 & 1.1 \\
\hline 23 & $37.878(7)$ & 2.75607 & $1,471.77$ & 224.77 & 2.05 \\
\hline 24 & 43.53(1) & 2.41234 & 1,103.77 & 140.12 & 1.28 \\
\hline 25 & $45.026(3)$ & 2.33616 & $1,037.09$ & 830.94 & 7.57 \\
\hline 26 & $45.284(2)$ & 2.32356 & $1,019.88$ & 594.49 & 5.42 \\
\hline 27 & $47.60(4)$ & 2.21678 & 905.75 & 49.94 & 0.46 \\
\hline 28 & 53.388(3) & 1.99121 & 788.36 & 348.09 & 3.17 \\
\hline 29 & $54.677(4)$ & 1.94777 & 763.61 & 326.05 & 2.97 \\
\hline 30 & 64.329(3) & 1.6803 & 595.76 & 240.83 & 2.19 \\
\hline
\end{tabular}

